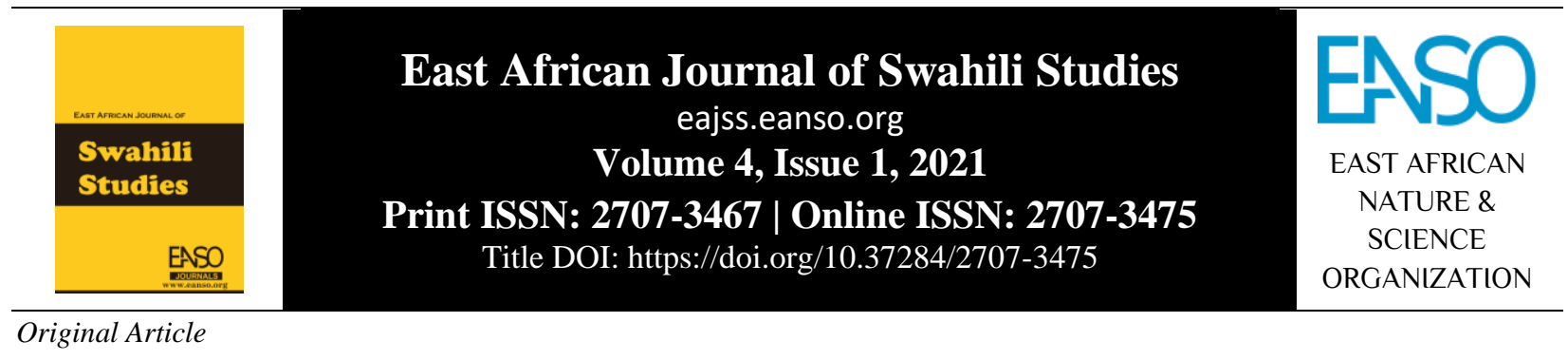

\title{
Nomino Ambatani za Kiswahili Zinazokiuka Kanuni ya Kufuta Mabano.
}

\author{
Mofart Onyoni Ayiega \& Leonard Chacha Mwita \\ ${ }^{1}$ Chuo Kikuu cha Kenyatta, S.L.P 43844-00100. Nairobi, Kenya. \\ *ORCID ID: https://orcid.org/0000-0001-7218-3991; Barua pepe ya mawasiliano: mofartayiega94@ gmail.com
}

DOI ya Nakala: https://doi.org/10.37284/eajss.4.1.452

\section{Tarehe ya Uchapishaji: IKISIRI}

30 Ocktoba 2021 Makala hii inachanganua nomino ambatani za Kiswahili zinazokiuka Kanuni ya Kufuta Mabano. Kanuni hii huonesha utaratibu wa kujenga neno kamili kwa

Istilahi Muhimu: kutumia vijenzi. Mabano katika kanuni hii hudhihirisha mipaka na hufutwa kuashiria kuwa sheria za uundaji wa maneno hazina ufikiaji wa muundo wa

Mofimu, maneno yaliyotokana na ngazi za awali. Utafiti huu ulifanywa baada ya kubaini

Mwambatano, kuwa baadhi ya nomino ambatani za Kiswahili hukiuka Kanuni ya Kufuta

Ukiukaji, Mabano hasa pale ambapo mabadiliko hutokea katikati mwa neno baada ya

Kanuni ya Kufuta

Mabano,

Mabadiliko,

Utaratibu. vipashio mbalimbali kuunganishwa. Nomino ambatani ifuatayo inadhihirisha mabadiliko yanayotokea katikati mwa neno baada ya vipashio mbalimbali kuunganishwa; [mw [enyekiti]] $\rightarrow$ [we [enyeviti]]. Katika utafiti huu tulikusanya data kutoka kwenye Kamusi Kuu ya Kiswahili (2015) ambapo nomino ambatani thelathini zinazokiuka Kanuni ya Kufuta Mabano zilitumika kama data ya msingi. Nomino hizi zilichaguliwa kwa kuzingatia sifa bainifu za ukiukaji wa Kanuni ya Kufuta Mabano (KKM). Utafiti huu uliongozwa na mhimili mmoja wa nadharia ya Mofolojia Leksia iliyoasisiwa na Kiparsky (1982) na kuendelezwa na Katamba na Stonham (2019): Kanuni ya Kufuta Mabano. Nadharia ya Mofolojia Leksia iliibuka kufidia mtazamo wa Chomsky wa Sarufi Zalishi ambao haukutambua kiwango cha mofolojia kama kiwango mahususi cha lugha. Kimsingi nadharia hii inaonesha uhusiano wa sheria zinazojenga maumbo ya kimofolojia na sheria zinazodhibiti namna maumbo hayo yanavyotamkwa. Data iliyohusiana na mada ya utafiti ilikusanywa maktabani. Baadhi ya makala ambazo zilitumika katika utafiti huu ni tasnifu za awali, makala ya mtandaoni na majarida. Utafiti huu uliongozwa na usampulishaji kimakusudi ili kufikia maneno husika ya nomino ambatani yaliyo na sifa bainifu. Matokeo ya utafiti huu yaliwasilishwa kwa njia ya michoro, majedwali na maelezo. Utafiti huu unachangia isimu hasa kupitia kuelewa na kueleza mbinu za mwambatano katika lugha ya Kiswahili,

30 | This work is licensed under a Creative Commons Attribution 4.0 International License. 
kuwahami waundakamusi za Kiswahili na maarifa, na kukuza mofolojia ya Kiswahili.

\begin{abstract}
APA CITATION
Ayiega, M. O. \& L. C. Mwita. (2021). Nomino Ambatani za Kiswahili Zinazokiuka Kanuni ya Kufuta Mabano. East African Journal of Swahili Studies, 4(1), 30-42. https://doi.org/10.37284/eajss.4.1.452.
\end{abstract}

\title{
CHICAGO CITATION
}

Ayiega, Mofart Onyoni \& Leonard Chacha Mwita. 2021. "Nomino Ambatani za Kiswahili Zinazokiuka Kanuni ya Kufuta Mabano". East African Journal of Swahili Studies 4 (1), 30-42. https://doi.org/10.37284/eajss.4.1.452.

\section{HARVARD CITATION}

Ayiega, M. O. \& L. C. Mwita (2021) “Nomino Ambatani za Kiswahili Zinazokiuka Kanuni ya Kufuta Mabano”, East African Journal of Swahili Studies, 4(1), pp. 30-42. doi: 10.37284/eajss.4.1.452.

\section{IEEE CITATION}

M. O. Ayiega \& L. C. Mwita, "Nomino Ambatani za Kiswahili Zinazokiuka Kanuni ya Kufuta Mabano", EAJSS, vol. 4, no. 1, pp. 30-42, Oct. 2021.

\section{MLA CITATION}

Ayiega, Mofart Onyoni \& Leonard Chacha Mwita . "Nomino Ambatani za Kiswahili Zinazokiuka Kanuni ya Kufuta Mabano". East African Journal of Swahili Studies, Vol. 4, no. 1, Oct. 2021, pp. 30-42, doi:10.37284/eajss.4.1.452.

\section{UTANGULIZI}

Mada ya makala hii inapatikana katika eneo jumla la mofolojia. Huu ni utanzu wa isimu unaoshughulikia muundo wa maneno (Habwe na Karanja, 2004). Haspelmath na Sims (2013) wanaeleza mofolojia kama utanzu wa isimu unaochunguza muundo wa ndani wa maneno. Kutokana na fasili hizi tunakubaliana na mawazo ya wataalam hawa kuwa mofolojia ni tawi la isimu ambalo hujihusisha na muundo wa maneno. Mofolojia kama taaluma imegawanywa katika matawi mawili makuu: mofolojia ya minyambuliko ya maneno na mofolojia ya uundaji wa maneno. Mofolojia ya minyambuliko ya maneno hushughulika na mchakato wa minyambuliko ya maneno ambayo huwekwa kwenye mizizi ya maneno kuwakilisha maana mbalimbali lakini minyambuliko hiyo haibadili aina ya neno hilo. Mofolojia ya uundaji wa maneno ni eneo ambalo hushughulikia mikondo na matokeo ya kiisimu ya uundaji wa maneno kwa kuweka viambishi katika mizizi ya maneno, na kiambishi nyambuaji huunda neno jipya (Mgullu, 1999; Katamba \& Stonham, 2019).

Uundaji wa maneno katika Kiswahili hufanyika kwa kutumia mbinu mbalimbali. Mbinu hizi huwa na utaratibu maalumu wa kuzalisha maumbo yenye sifa tofauti. Mbinu hizo ni kama; utohozi, ufupishaji (akronimu, uhulutishaji na mkato), upanuzi wa maana ya maneno, toponimu, eponimu na mwambatano (Rubanza, 1996; Buberwa, 2011; Matinde, 2012).

Mbinu ya mwambatano hutumia maneno kamili yaliyoundwa kwa kutumia njia mbalimbali kama malighafi ya kuunda maneno mengine mapya kabisa. Maumbo yanayozalishwa huandikwa kwa kuunganisha maneno mawili, kwa kutumia vistari au kuacha nafasi kati ya maneno yaliyoambatanishwa (Gichuru, 2010). Hata hivyo, katika makala hii tuliamua kuandika nomino ambatani hizi katika namna mbili yaani; kuunganisha maneno yote na kutenganisha baadhi ya maneno katika nomino ambatani hizi ili kurahisisha uchanganuzi wa data uliofanyika. Mgullu (1999) anatazama nomino ambatani kama mofu changamano. Anasema kuwa mofu changamano ni mofu ambazo huundwa kwa kuweka pamoja angalau mashina au mizizi miwili ya maneno ambayo katika mazingira mengine mashina hayo yanaweza kutenganishwa na kila moja likawa ni neno kamili. Maoni ya Mgullu (keshatajwa) yanayooana na yale ya Mustafa (2020) ambaye anashikilia huwa nomino ambatani, ni nomino zilizoundwa kwa kuunganisha maneno mawili (mizizi, kiini, mofimu huru au viambatisho vya leksia). Maneno mawili haya huweza kuwa na nafasi, bila nafasi au kistariungio. Mathalan kama inavyodhihirika katika nomino ambatani 'mwanamke' na 'mwalimu mkuu.' 


\section{Tatizo Linalotafitiwa}

Kanuni ya Kufuta Mabano ni mhimili ambao hutumiwa kuonesha utaratibu wa kujenga neno kamili kwa kutumia vijenzi vyake. Mabano katika kanuni hii, huashiria mipaka ya vipashio mbalimbali na hufutwa kuonesha kuwa sheria za uundaji wa maneno hazina ufikiaji wa muundo wa maneno yaliyotokana na ngazi za awali (Katamba \& Stonham, 2019).

Kanuni ya Kufuta Mabano huwa na mkabala ambao unasisitiza kuwa mabano ya katikati huwa ya kwanza kufutwa katika kiwango cha leksia. Kutokana na matokeo ya mkabala huu, maneno yanayoingiliana katikati mwa ngazi leksia hayawezi kuathiriwa na sheria za fonolojia mzunguko (Kiparsky, 1982; Katamba na Stonham, 2019).

Baada ya kufuta mabano, maneno yanayotokana na mofolojia na sheria za uundaji wa maneno hazina ufikiaji wa muundo wa ndani wa maneno. Mkabala huu unaruhusu urejeleaji wa nje wala si ndani ya neno baada ya mabano kufutwa. Mofimu ya kwanza katika neno zima huweza kuwekwa katika hali ya wingi bila kuingia katika mofimu ya pili katika neno zima (Allen, 1978; Siegel, 1974). Mofimu ya kwanza tunayorejelea hapa ni kiambishi cha ngeli kinachopatikana katika neno la kwanza la nomino ambatani, na mofimu ya pili ikiwa ni sehemu ya nomino ambatani inayobeba mzizi au shina la neno la kwanza pamoja na neno la pili au tatu katika neno ambatani, mathalan nomino ambatani 'mwanamke' ina mofimu mbili ambazo ni $\{m w\}$ ikiwa mofu ya kwanza na mofimu ya pili (anamke). Nomino ambatani kulingana na Kanuni ya Kufuta Mabano huwa na utaratibu ufuatao:

$$
\mathrm{Y}_{1}+\mathrm{Y}_{2} \rightarrow\left[\left[\mathrm{Y}_{1}\right]\left[\mathrm{Y}_{2}\right]\right] \rightarrow\left[\mathrm{Y}_{1} \mathrm{Y}_{2}\right]
$$

$\left[[\mathrm{mw}]_{\text {kia. }}[\text { ana }]_{\text {mzizi }}+\left[[\mathrm{m}]_{\text {kia. }}[\mathrm{ke}]_{\text {mzizi }}\right]_{\mathrm{N}} \rightarrow[[\right.$ mwana $]$ $[$ mke $]]_{\mathrm{N}} \rightarrow[\text { mwanamke }]_{\mathrm{N}}$ Mwanamke

Haua 1

Hatua 2

Hatua 3

Neno ambatani kama hili lililo hapo juu linapoundwa na kufikia hatua ya 3 linakuwa na mofimu mbili tu, yaani $\{\mathrm{mw}\}+\{$ anamke $\}$ kinyume na ilivyokuwa katika hatua ya 1 ambapo lilikuwa na mofimu $\left\{\{\mathrm{mw}\}_{\text {kia. }}\{\text { ana }\}_{\text {mzizi }}+\left\{\{\mathrm{m}\}_{\text {kia. }}\{\mathrm{ke}\}_{\text {mzizi }}\right\}\right.$. Kwa mujibu wa Kanuni ya Kufuta Mabano, katika hatua ya 3 huwezi kutenga $\{$ ana $\}$ na $\{$ mke $\}$ na hivyo basi hakuna mabadiliko yanayostahili kutokea katika mofimu hii. Katika wingi, nomino ambatani mwanawake inakuwa wanawake ilhali tunajua kuwa katika umoja tuna mofimu mbili $\{\mathrm{mw}\}$ na \{anamke\}, mabadiliko haya katikati mwa mofimu \{anamke\} baada ya vipashio mbalimbali kuunganishwa huwa ni jambo la kushangaza kwani Kanuni ya Kufuta Mabano hairuhusu mabadiliko kutokea katikati mwa neno baada ya vipashio mbalimbali kuunganishwa. Baadhi ya nomino ambatani za Kiswahili hukiuka utaratibu huu na hili ndilo tatizo linalotafitiwa.

Makala hii ilichunguza baadhi ya nomino ambatani za Kiswahili ambazo zinakiuka Kanuni ya Kufuta Mabano (KKM) kwa kuchukua viambishi vya mofimu za wingi katika maneno yote yanayounda nomino ambatani husika ambayo ni kinyume na Kanuni ya Kufuta Mabano inayoruhusu uchukuaji wa mofimu za wingi katika mofimu ya kwanza pekee katika neno husika. Kutoa mwanga zaidi kuhusu kile tunachorejelea hapa tutaorodhesha nomino ambatani zinazokubali mabadiliko kutokea katikati mwa neno na zile zisizokubali mabadiliko kutokea kama inavyodhihirka katika Jedwali 1 lifuatalo:

Jedwali 1: Nomino Ambatani Zinazokiuka na Zisizokiuka Kanuni ya Kufuta Mabano

\begin{tabular}{lll}
\hline $\begin{array}{l}\text { Nomino Ambatani za Mabadiliko Kutokea } \\
\text { Katikati mwa Neno }\end{array}$ & $\begin{array}{l}\text { Nomino Ambatani Zisizo na Mabadiliko } \\
\text { Kutokea Katikati mwa Neno }\end{array}$ \\
\hline [Mw [anamke]] - [wa [anawake]] & {$[$ Mw [anasiasa]] - [w [anasiasa]] } \\
[M [kazamjomba]] - [wa [kazawajomba]] & {$[$ Ch [amshakinywa]] - [vi [amshakinywa]] } \\
{$[$ Mw [anamwali]] - [w [anawali]] } & {$[$ Ki [pandesauti]] - [vi [pandesauti]] }
\end{tabular}

Uundaji wa maneno ni swala muhimu katika isimu na hudhibitiwa na kanuni na utaratibu wa sarufi ya lugha husika. Mada hii ilichaguliwa ili kuchangia katika kueleza na kuelewa Kanuni ya Kufuta Mabano ya Mofolojia Leksia katika uundaji wa nomino ambatani za Kiswahili. Nomino ambatani 
zilichaguliwa kutokana na pengo lililodhihirika na ambalo tafiti za Kiswahili hazikuwa zimetekeleza. Utafiti huu ulikuwa muhimu katika kubainisha ukiukaji wa KKM katika uundaji wa nomino ambatani za Kiswahili. Mada hii ni muhimu katika Kiswahili kwa sababu itawaongoza wanaleksikografia kufahamu vighairi vya Kanuni ya Kufuta Mabano ya Mofolojia Leksia ambavyo huchukua viambishi vya mofimu za wingi katika mofimu zote za nomino ambatani kinyume na KKM ambayo hairuhusu mabadiliko katikati mwa neno baada ya vipashio mbalimbali kuunganishwa.

\section{Tafiti za Awali}

Usomaji mpana wa makala maktabani, vitabu, na makala ya mtandao ulinuiwa kupata taarifa muhimu kuhusu ukiukaji wa KKM ya Mofolojia Leksia katika uundaji wa nomino ambatani za Kiswahili na pia kuelewa mbinu ya mwambatano katika lugha ya Kiswahili. Maelezo yaliyopatikana yalikuwa na mashiko katika kubainisha pengo linaloshughulikiwa na utafiti huu.

Gituru (2019) katika utafiti wake juu ya unyambulishaji wa vitenzi katika Kigĩchũgũ, alibaini mifanyiko na mfuatano wa vitenzi vya Kigĩchũgũ katika mchakato wa unyambuzi kwa kuongozwa na nadharia ya Mofolojia Leksia na Kanuni ya Kioo. Aidha katika uchunguzi huu alidhihirisha kuwa Kanuni ya Kufuta Mabano huonesha mageuzi ya vitenzi yanayotokea katika mchakato wa unyambuzi, na maumbo mbalimbali huungana kuwa kitenzi kimoja. Utafiti huu ulikuwa msingi wa kueleza utaratibu wa Kanuni ya Kufuta Mabano katika mchakato wa uundaji maneno na maelezo zaidi kuhusu nadharia ya Mofolojia Leksia.

Odden (2019) katika uchunguzi wake kuhusu kutabiri toni katika Kikuria, alitambua kuwa Kanuni ya Kufuta Mabano hukiukwa katika uwekaji wa toni katika vitenzi vya Kikuria hasa sheria ya kuhamishwa kwa shina inapokuwa wazi kwa mabano ya ndani ya shina. Anaendelea kusema kuwa hali hii hutokea wakati toni huhamishwa kutoka kiambishi kwenda shina la kitenzi cha Kikuria na hivyo basi, hukiuka KKM ambayo hairuhusu fonolojia kurejelea mofolojia ya ndani ya neno. Maarifa kutoka utafiti huu, yalitufaa katika kuelewa mchakato mzima wa uundaji wa nomino ambatani na namna baadhi za nomino hizi hukiuka
Kanuni ya Kufuta Mabano kwa kuweka wazi mofolojia ya ndani ya neno kwa fonolojia.

Katikiro (2018) alitafiti kuhusu tathmini ya maana katika maneno ambatani ya Kiswahili. Katika utafiti huo alishughulikia ubainishaji wa mchango wa maana za manenojenzi katika maana tokeo ya neno ambatani. Akirejelea Gordian (2010), alidai kuwa maneno ambatani hutokana na mchakato wa kimofolojia wa kuambatisha maneno mawili yaliyo tofauti kimaana na kiumbo ili kuunda neno moja jipya lenye maana moja au lenye kurejelea kitu au dhana moja. Katika utafiti huu, Katikiro (2018) alijikita katika uchunguzi wa tawi la semantiki ambalo hujihusisha na maana katika tungo tofauti ambazo ni kinyume na utafiti tuliotekeleza ambao ulijihusisha na tawi la isimu la mofolojia. Hata hivyo, maarifa kutoka utafiti huu yalitufaa katika kufahamu mchakato wa kimofolojia wa uundaji nomino ambatani kwa kuambatisha maneno mawili yaliyo tofauti kimaana na kiumbo ili kuunda dhana moja.

Buberwa (2017) akichunguza ruwaza za kimofolojia za majina ya mahali katika lugha ya Kihaya, alitambua mpangilio wa vipashio vinavyounda majina hayo na kuonesha jinsi vipashio vilipangana kimsonge kwa kuongozwa na mhimili wa Kanuni ya Ngazi Leksia ya Mofolojia Leksia. Katika utafiti huo alieleza nadharia ya Mofolojia Leksia kwa uwazi. Hata hivyo, tofauti kuu kati ya utafiti wa Buberwa (2017) na utafiti huu ni kuwa utafiti wake ulichunguza mpangilio wa maneno ya Kihaya na kuonesha jinsi vipashio vinapangwa kimsonge kwa kuongozwa na mhimili wa Kanuni ya Ngazi Leksia ya Mofolojia Leksia. Kwa upande mwingine, utafiti tuliotekeleza ulichunguza nomino ambatani za Kiswahili zinazokiuka KKM ya Mofolojia Leksia. Utafiti huu ulitufaa katika kueleza dhana ya nadharia ya Mofolojia Leksia.

Buberwa (2011) katika utafiti wake kuhusu muundo wa majina ya mahali, alibaini mbinu za uundaji nomino kama vile; kuhamisha maana, toponimu, eponimu na mbinu ya mwambatano, zinazotumika katika kuunda majina ya vituo vya daladala jijini Dar es Salaam. Utafiti huu ulikuwa na mshabaha na wetu kwa kuwa ulishughulikia mbinu ya mwambatano, hata hivyo, ni tofauti kwani utafiti wetu ulichunguza nomino ambatani za Kiswahili 
ambazo hukiuka KKM. Maarifa kutoka utafiti huu yalitusaidia katika kuelewa dhana ya mwambatano ambayo utafiti tuliotekeleza ulichunguza.

Gichuru (2010), akichunguza nomino ambatani za Kiswahili, alibainisha na kuainisha taratibu pamoja na kanuni zinazohusika katika uundaji wa nomino ambatani kwa kuongozwa na mihimili sita ya nadharia ya Mofolojia Leksia. Utafiti wake ulidondoa data yake kutoka Nyuso za Mwanamke (2010), Kamusi ya Isimu na Falsafa ya Lugha (2004) na Kamusi ya Kiswahili Sanifu (2004). Utafiti huo ulitilia mkazo maumbo ya nomino ambatani, kanuni na taratibu zilizohusika katika kuunda nomino ambatani na uhusiano wa kisarufi uliopo baina ya vijenzi vya nomino ambatani. Ni ukweli kwamba utafiti wa Gichuru (2010) ulikuwa na mshabaha kiasi na utafiti tuliotekeleza kwani tafiti zote zilijikita katika aina moja ya data ambayo ni nomino ambatani na nadharia ya Mofolojia Leksia.

Hata hivyo, tafiti hizi zina tofauti kwani Gichuru (2010) alijikita katika uchanganuzi wa uundaji wa nomino ambatani za Kiswahili na uambishaji wake kisarufi kwa kuongozwa na mihimili sita ya Mofolojia Leksia (Kanuni ya Mzunguko Kamili, Kanuni ya Ngazi Leksia, Kanuni ya Kufuta Mabano, Kanuni ya Kuhifadhi Muundo, Kanuni ya Ufinyu wa Sifa na Kanuni ya Kwingineko ya Mofolojia Leksia) ilhali utafiti huu ulihusika na kuchunguza nomino ambatani zilizokiuka Kanuni ya Kufuta Mabano kwa kuchukua mofimu za wingi kwa maneno yote yanayounda neno husika. Maarifa kuhusu nomino ambatani na nadharia ya Mofolojia Leksia kutoka kwa utafiti huu yalikuwa ya kufaa katika utafiti wa mada hii teule.

Orgun na Inkelas (2002) walieleza kuwepo kwa sababu za kutazama tena ufutaji mabano. Katika utafiti wao walieleza kuhusu ufutaji wa mabano ambapo waligundua kuwa ufutaji mabano haujapewa uzingativu mwingi katika mofolojia ya maneno. Walidhihirisha kauli hii kwa kurejelea Pesetsky (1979) ambaye alieleza kuwa mkabala wa kufuta mabano unaruhusu fonolojia kurejelea mipaka iliyotolewa na ujenzi wa mofolojia wa nje, lakini hauruhusu urejeleaji wa muundo wa ndani ulioingia zaidi. Maarifa ya Orgun na Inkelas (2002) yalifaa utafiti huu kwa kuwa yalikuwa ni kichocheo cha kung'amua jinsi nomino ambatani za Kiswahili hukiuka Kanuni ya Kufuta Mabano.

Kiparsky (1982) alieleza kuhusu mkabala wa kufuta mabano ambao unasisitiza kwamba mabano ya katikati huwa ya kwanza kufutwa katika kiwango cha leksia. Kama matokeo ya mkabala huu, maneno yanayoingiliana katikati mwa ngazi leksia hayawezi kuathiriwa na sheria za fonolojia mzunguko. Baada ya kufuta mabano, maneno yanayotokana na mofolojia huchukuliwa kana kwamba yalibuniwa. Kwa maoni ya Kiparsky (1982), utasa huu unaenea kwa michakato ya mofolojia na sheria za uundaji wa maneno hazina ufikiaji wa muundo wa ndani wa maneno yaliyotokana na kiwango cha awali. Maarifa haya yalifaa katika utafiti uliotekelezwa.

Akitafiti kuhusu kuondoa mzunguko kama chanzo cha ugumu wa fonolojia, Cole (1995) alitambua mofolojia ya ndani ya neno kuwa wazi kwa fonolojia hata baada ya neno kuundwa kikamilifu. Anaendelea kusema kuwa lazima muundo wa ndani wa mofolojia ya neno uonekane ili kupata athari za mzunguko kwa kuweka vizuizi katika vikoa vilivyoainishwa kimofolojia. Kutokana na uchunguzi huu, tulipata maarifa jinsi mofolojia ya ndani huonekana kwa fonolojia, hali ambayo ni kinyume na Kanuni ya Kufuta Mabano inayoruhusu fonolojia kurejelea muundo wa nje wa neno. Hili lilitufaa kutambua jinsi baadhi za nomino ambatani za Kiswahili hukiuka KKM kwa kuruhusu fonolojia kuangalia muundo wa ndani wa nomino husika baada ya vipashio mbalimbali kuunganishwa.

Mapitio haya ya maandishi yamedhihirisha kuwa ingawa kuna tafiti ambazo zimefanyika kuhusiana na mbinu ambatani ya kuunda nomino za Kiswahili, ukiukaji wa Kanuni ya Kufuta Mabano haujawekwa bayana. Kwa hivyo, makala hii inadhihirisha baadhi za nomino ambatani za Kiswahili zinazokiuka Kanuni ya Kufuta Mabano hasa zinapokubali mabadiliko kutokea katikati mwa neno baada ya vipashio mbalimbali kuunganishwa.

\section{Misingi ya Nadharia}

Makala hii iliongozwa na nadharia ya Mofolojia Leksia (ML) iliyoasisiwa na Kiparsky (1982) na kuendelezwa na Katamba na Stonham (2019). Nadharia ya ML iliibuka kufidia mtazamo wa Chomsky wa Sarufi Zalishi ambao haukutambua kiwango cha mofolojia kama kiwango mahususi cha 
lugha. Kimsingi nadharia hii inaonesha uhusiano wa sheria zinazojenga maumbo ya kimofolojia na sheria zinazodhibiti namna maumbo hayo yanavyotamkwa (Kiparsky, 1982; Katamba na Stonham, 2019). Sheria katika leksia zimepangwa kwenye ngazi kimsonge (Kiparsky, 1982; Katamba na Stonham, 2019; McMahon, 2000). Hii huashiria kwamba viambishi (mofimu) vinavyopachikwa katika mzizi ili kuunda neno hupangwa kidaraja.

Nadharia hii imetambua viwango viwili vya kanuni; kanuni leksika ambazo hujihusisha na kuunda neno kubalifu kileksika na kanuni kirai kwa upande mwingine huhusika na utendaji kazi baada ya neno kuundwa. Mihimili ya nadharia hii ni sita yaani Kanuni ya Ngazi Leksia, Kanuni ya Mzunguko Kamili, Kanuni ya Ufinyu wa Sifa, Kanuni ya Kwingineko, Kanuni ya Kuhifadhi Muundo, na Kanuni ya Kufuta Mabano. Makala hii iliongozwa na mhimili mmoja: Kanuni ya Kufuta Mabano ambao huonesha utaratibu wa kujenga neno kamili kwa kutumia vijenzi vyake. Kanuni hii hushukilia kuwa wakati sheria ya ngazi moja ya kifonolojia imetumika, mabano yake hufutwa mpaka mwishowe neno lenye umbo kamili linapatikana kama inavyojitokeza katika nomino ambatani 'mwenyekiti' ifuatayo:

$[\text { Enye }]_{\mathrm{V}}[\mathrm{kiti}]_{\mathrm{N}}$

Hatua 1

$\downarrow$

$\left.[[\mathrm{Mw}][\mathrm{enye}]]_{\mathrm{V}}[\mathrm{kiti}]_{\mathrm{N}}\right]_{\mathrm{N}} \quad$ Hatua 2

$$
\downarrow
$$

$[\text { Mwenyekiti }]_{\mathrm{N}}$

Hatua 3

\section{Mwenyekiti}

Hatua 4

Kinachodhihirika hapa juu ni kuwa vipashio mbalimbali huunganishwa kuanzia hatua ya kwanza mpaka hatua ya tatu ambapo mabano hubomolewa baada ya neno kuundwa ambayo ni hatua ya nne na mwisho katika utaratibu wa kuunda nomino ambatani.

Katika utafiti tuliotekeleza tulibainisha kuwa baadhi za nomino ambatani hukiuka Kanuni ya Kufuta Mabano hasa zinapochukua mofimu za wingi kwa

maneno yote yanayounda neno husika kama utaratibu ufuatao unavyoonesha:

$$
\left[\text { wingi }+\mathrm{Y}_{1}\right]+\left[\text { wingi }+\mathrm{Y}_{\mathrm{n}}\right]
$$

Maelezo: Kijenzi tangulizi (neno kuu) pamoja na vijenzi vingine $\left(Y_{n}\right)$ vinaongezwa viambishi vya kiidadi katika upande wa kushoto wa kila kijenzi (Gichuru, 2010).

Kinachodhihirika wazi kutokana na maelezo ya hapo juu ni kuwa maneno yote yanayounda nomino husika yanabadilika katika wingi kinyume na Kanuni ya Kufuta Mabano ya Mofolojia Leksia ambayo hushikilia kuwa baada ya mabano kufutwa sheria za kimofolojia haziwezi kurejelea hali za ndani za muundo wa kimofolojia wa awali. Ufuatao ni mfano wa nomino ambatani inayokiuka Kanuni ya Kufuta Mabano:

\section{Umoja}

[Mw [enyekiti]]

Hapa kinachodhihirika ni kuwa uambishaji kisarufi una uwezo wa kuangalia nyuma, kinyume na Kanuni ya Kufuta Mabano inayoshikilia kuwa uambishaji wa kisarufi hauna uwezo wa kuangalia nyuma na iwapo utaangalia nyuma huchukua mofimu za wingi katika mofimu ya kwanza pekee na hauruhusu mabadiliko katikati mwa neno baada ya vipashio mbalimbali kuunganishwa. Mhimili huu ulitusaidia katika kuchanganua data ya nomino ambatani zilizokiuka KKM kwa kukubali mabadiliko kutokea katikati mwa neno baada ya vipashio mbalimbali kuunganishwa.

\section{Mbinu za Utafiti}

Data ya utafiti huu ilikusanywa kutoka Kamusi Kuu ya Kiswahili (2015). Vilevile majarida na makala yanayohusiana na mada ya utafiti huu yalizingatiwa. Mtafiti alitumia sampuli ya kimakusudi kuteua nomino ambatani za Kiswahili kutoka Kamusi Kuи ya Kiswahili (2015). Kutokana na utafiti wa awali, nomino ambatani 30 pekee katika Kamusi Kии ya Kiswahili (2015) hukiuka KKM. Sababu ya kuteua maneno 30 pekee katika utafiti tuliotekeleza ni kutokana na kuongozwa na maoni ya Milroy (1987) ambaye anapendekeza matumizi ya sampuli ndogo katika utafiti wa lugha kwa kuwa sampuli kubwa inaweza kuzua data 
yenye urudufu mwingi na pia data chache haifai katika utafiti. Kutokana na maoni ya Milroy (1987) ni wazi kuwa data wastani ilifaa katika utafiti huu.

\section{Matokeo ya Utafiti Huu}

Makala hii imeshughulikia nomino ambatani za Kiswahili ambazo zinakiuka Kanuni ya Kufuta Mabano kwa kuruhusu mabadiliko kutokea katikati mwa neno baada ya vipashio mbalimbali kuunganishwa. Mabadiliko haya hutokea pale ambapo mofimu ya pili katika nomino ambatani huchukua viambishi vya wingi, hali ambayo ni kinyume na Kanuni ya Kufuta Mabano inayoruhusu fonolojia kurejelea mipaka ya nje kabisa iliyowekwa katika uundaji wa kimofolojia wa neno.
Kanuni hii hushikilia kuwa baada ya mabano ya katikati kufutwa sheria za uundaji wa maneno hazina ufikiaji wa muundo wa ndani wa maneno. Lengo kuu katika makala hii, ni kudhihirisha nomino ambatani za Kiswahili zinazokiuka Kanuni ya Kufuta Mabano kwa kuongozwa na mhimili mmoja; Kanuni ya Kufuta Mabano ya Mofolojia Leksia (Kiparsky, 1982).

\section{Nomino Ambatani za Kiswahili Zinazokiuka Kanuni ya Kufuta Mabano}

Katika utafiti tuliotekeleza maktabani, tulibaini nomino ambatani thelathini ambazo zinakiuka Kanuni ya Kufuta Mabano ambazo zimeorodheshwa katika Jedwali 2.

\section{Jedwali 2: Nomino Ambatani Zinazokiuka Kanuni ya Kufuta Mabano}

\begin{tabular}{|c|c|}
\hline Nomino ambatani (Umoja) & Nomino Ambatani (Wingi) \\
\hline $\begin{array}{l}\text { [Mw [anamke]] } \\
{[\mathbf{M} \text { [jamzito]] }} \\
[\emptyset \text { [raia mwema }]] \\
{[\text { Mw [alimu mkuu]] }} \\
\text { Nomino ambatani (Umoja) }\end{array}$ & $\begin{array}{l}\text { [wa [anawake]] } \\
{[\mathbf{w a}[\text { jawazito]] }} \\
\text { [ø [raia wema]] } \\
\text { [w [alimu wakuu]] } \\
\text { Nomino Ambatani (Wingi) }\end{array}$ \\
\hline 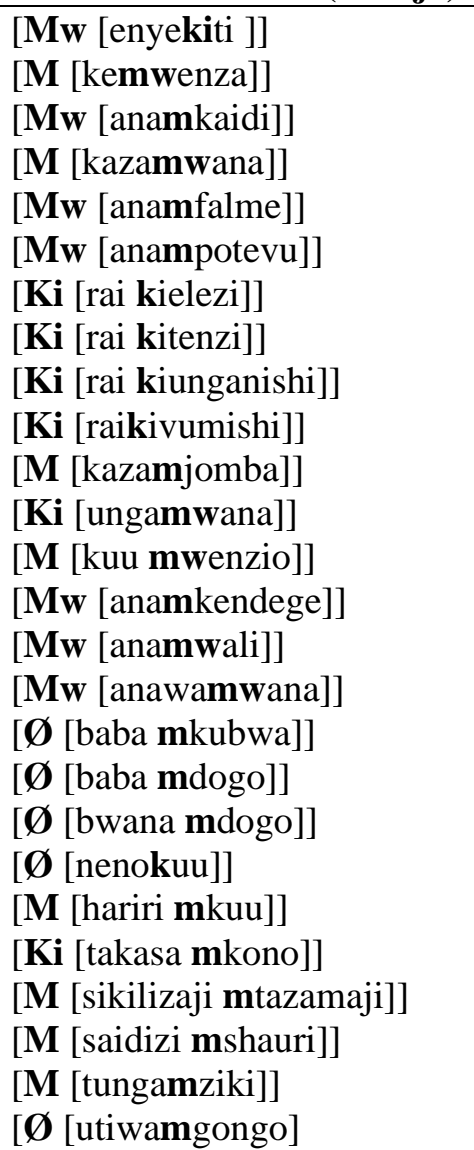 & 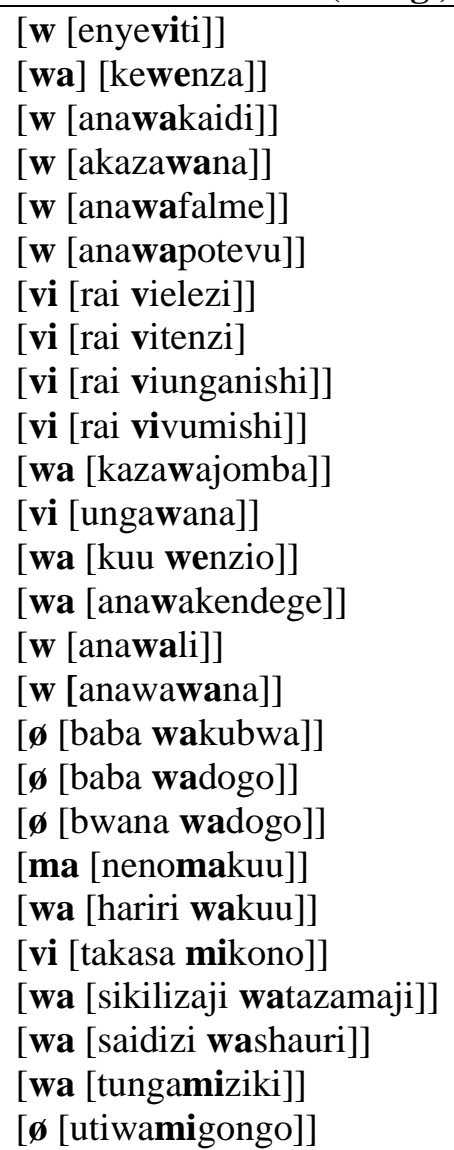 \\
\hline
\end{tabular}

36 This work is licensed under a Creative Commons Attribution 4.0 International License. 
Data ya nomino ambatani tulizoorodhesha katika Jedwali 2 inadhihirisha ukiukaji wa Kanuni ya Kufuta Mabano. Kanuni hii, huwa na mkabala ambao unasisitiza kuwa mabano ya katikati huwa ya kwanza kufutwa katika kiwango cha leksia na iwapo urejeleaji utakuwa katika nomino ambatani, urejeleaji huu huwa katika mofimu ya kwanza pekee (Allen, 1978; Kiparsky, 1982; Katamba na Stonham, 2019). Kutoa mwanga zaidi kuhusiana na ukiukaji wa Kanuni ya Kufuta Mabano, tutaeleza utaratibu wa uundaji wa nomino ambatani 'mjamzito' kutoka Jedwali 2 kama kiwakilishi cha nomino zingine ambatani 29 zilizoorodheshwa hapa juu kama ifuatavyo katika Kielelezo 1:

\section{Kielelezo 1: Utaratibu wa Uundaji Nomino Ambatani 'Mjamzito'}

$\left[\left[[\mathrm{m}]_{\text {kia }}[\mathrm{ja}]_{\text {shina }}\right]\left[[\mathrm{m}]_{\text {kia. }}[\text { zito }]_{\text {shina }}\right]\right] \quad$ Hatua 1

$\left[\left[\mathrm{mja}_{\mathrm{N}}[\mathrm{mzito}]\right]_{\mathrm{v}} \quad\right.$ Hatua 2

$[\text { mjamzito }]_{\mathrm{N}} \quad$ Hatua 3

Mjamzito $\rightarrow{ }^{*}$ wajamzito (sio sahihi)

$$
\text { Wajawazito (sahihi) }
$$

Kinachodhihirika katika utaratibu huu ni kuwa hatua ya kwanza inajihusisha na kutenganisha vipashio mbalimbali kwa kutumia mabano. Mathalan katika hatua hii viambishi katika nomino ambatani 'mjamzito' vimetenganishwa na shina $\left[\left[[\mathrm{m}]_{\text {kia }}[\mathrm{ja}]_{\text {shina }}\right]\right.$ na $\left.\left[[\mathrm{m}]_{\text {kia. }}[\text { zito }]_{\text {shina }}\right]\right]$. Katika hatua ya pili, mabano ya katikati hufutwa kuonesha viambajengo vya nomino ambatani; [[mja $]_{\mathrm{N}}$ [mzito]]v, na katika hatua ya tatu nomino ambatani huwa tayari imeunganishwa; [mjamzito] $]_{N}$ na mabano hubomolewa kuonesha mwisho wa utaratibu wa uundaji wa nomino ambatani. Baada ya kuondoa mabano, maneno yaliyoundwa kwa kuunganisha vijisehemu kimofolojia huchukuliwa kama ambayo hayajaunganishwa, yamekuwa mazima hivyo tangu awali.

Kwa mujibu wa Orgun na Inkelas (2002), mabano yafutwapo katika mchakato wa uundaji wa nomino ambatani hii, Kanuni ya Kufuta Mabano hairuhusu fonolojia kurejelea maumbo yaliyokuwa ndani mathalan mofimu ya pili \{jamzito\}. Uambishaji kisarufi wa nomino ambatani 'mjamzito' katika hali ya wingi unastahili kuwa katika mofimu ya kwanza yaani, $\{m\}$ wala si katika mofimu ya pili \{jamzito\}. Kulingana na Kanuni ya Kufuta Mabano, uambishaji kisarufi wa nomino ambatani katika hali ya wingi unafaa kuwa *wajamzito, nomino ambayo huleta utata wa maana kisarufi. Kutoa utata huu wa maana kisarufi, nomino hii huruhusu uambishaji wa kisarufi katika mofimu ya pili yaani, \{jawazito hali ambayo husababisha mabadiliko kutokea katikati mwa nomino ambatani mjamzito baada ya vipashio mbalimbali kuunganishwa na kuwa wajawazito. Hali hii hutokea katika nomino ambatani zote tulizoorodhesha katika Jedwali 2, yaani nomino hizi huchukua viambishi vya wingi katika mofimu ya pili ya nomino ambatani. Hata hivyo, Orgun na Inkelas (2002) wanadai kuzuka kwa nadharia inayoweka wazi mofolojia ya ndani kwa fonolojia hata baada ya neno kuundwa kikamilifu yaani nadharia ya Umbo Upeo.

Nadharia ya Umbo Upeo hushikilia kuwa habari zote za mofolojia huonekana na fonolojia (Prince na Smolensky, 1993). Maoni haya yanaoana na ya Cole (1995) ambaye anasema kuwa muundo wa ndani wa mofolojia ya neno lazima uonekane ili kupata athari za mzunguko kwa kuweka vizuizi katika vikoa vilivyoainishwa kimofolojia. Maoni ya Orgun na Inkelas (2002) na Cole (1995) yanatofautiana na wataalam Siegel (1974), Allen (1978), Pesetsky (1979), Kiparsky (1982), Mohanan (1986), Inkelas (1990), Booij (1997) na Katamba \& Stonham (2019) ambao wanashikilia kuwa baada ya mabano kufutwa katika mchakato wa uundaji wa neno, Kanuni ya Kufuta Mabano huruhusu fonolojia kurejelea mipaka ya nje kabisa iliyowekwa katika uundaji wa kimofolojia lakini hairuhusu kurejelea maumbo yaliyokuwa ndani. Katika utafiti huu, tumedhihirisha baadhi za nomino ambatani za Kiswahili zinazokiuka Kanuni ya Kufuta Mabano kwa kuruhusu mabadiliko kutokea katika mofimu ya pili ya nomino ambatani tulizoorodhesha katika Jedwali 2 ambapo zinachukua viambishi vya wingi, mathalan: mofimu za nomino ambatani 'mkemwenza,' yaani mofimu ya kwanza [m] na mofimu ya pili [kemwenza]] huchukua viambishi vya wingi kuwa [wa [kewenza]].

Orgun na Inkelas (keshatajwa) wanaeleza namna ukiukaji wa Kanuni ya Kufuta Mabano 
unavyodhihirika katika lugha ya Kijapani ambapo fonolojia inaonekana kuhitaji ufikiaji zaidi wa muundo wa ndani wa neno kuliko vibali vya Kanuni ya Kufuta Mabano vinavyoruhusu fonolojia kurejelea mipaka ya nje iliyowekwa katika uundaji wa kimofolojia. Maoni ya Orgun na Inkelas (2002) yanaoana na Poser (1990) ambaye anadai kuwa shadda katika nomino za Kijapani zinazoundwa kutoka kwa vitenzi huonesha mfano wa kukomesha Kanuni ya Kufuta Mabano na sheria ya fonolojia inayotumiwa kwa unominishaji wa umbotokeo inahitaji kujua unominishaji wa pembejeo ulikuwa ambatani. Poser (keshatajwa) anaendelea kusema kuwa lazima sheria inayoweka shadda katika

Jedwali 3: Uwekaji Upya wa Shadda katika Nomino Ambatani za Kiswahili

\begin{tabular}{|c|c|}
\hline Uwekaji wa Shadda kila Neno & Uwekaji Upya wa Shadda \\
\hline 'Mja + 'mzito $\rightarrow$ *'mjam'zito & mjam'zito \\
\hline 'Mwana + 'mke $\rightarrow$ *'mwana'mke & mwana'mke \\
\hline 'Mwana + m'falme $\rightarrow{ }^{*}$ 'mwanam'falme & mwanam'falme \\
\hline M'kaza + m'jomba $\rightarrow$ "'m'kazam'jomba & mkazam'jomba \\
\hline $\begin{array}{l}\text { Msikili'zaji }+ \text { mtaza'maji } \rightarrow{ }^{*} \text { msikili'zaji } \\
\text { mtaza'maji }\end{array}$ & msikilizaji mtaza'maji \\
\hline M'tunga + m'ziki $\rightarrow{ }^{*}$ m'tungam'ziki $^{\prime}$ & mtungam'ziki \\
\hline Msai'dizi + msha'uri $\rightarrow{ }^{*}{ }^{*}$ sai'dizi msha'uri & msaidizi msha'uri \\
\hline M'kaza + 'mwana $\rightarrow{ }^{*}$ m'kaza'mwana & mkaza'mwana \\
\hline 'Mwana + 'mke + 'ndege $\rightarrow{ }^{*}$ 'Mwana'mke'ndege & mwanamke'ndege \\
\hline
\end{tabular}

Kinachotokea katika Jedwali 3 ni kuwa kila neno huwa na shadda ya msingi kabla ya kuunganishwa kuwa ambatani. Katika mchakato wa uwekaji upya wa shadda katika nomino ambatani, shadda huwekwa kwenye silabi ya pili kutoka mwisho katika nomino ambatani ambayo kimsingi inapatikana katika neno la pili la hapo mwanzoni kabla maneno hayo yaungane. Tutatumia 'mkazamjomba' kama kiwakilishi cha nomino ambatani tulizoorodhesha katika Jedwali 3 kueleza namna uwekaji upya wa shadda hutokea katika nomino ambatani. Kielelezo 2 kifuatacho kinadhihirisha jinsi shadda huwekwa katika utaratibu wa uundaji wa nomino ambatani 'mkazamjomba':

\section{Kielelezo 2: Uwekaji Upya wa Shadda katika 'Mkazamjomba'}

- Maneno mawili: mkaza + mjomba

- Shadda katika kila neno: m'kaza + m'jomba nomino zinazoundwa kutoka vitenzi itaje mpaka wa uambatani katika mazingira yake. Wanachomaanisha Orgun na Inkelas (2002) na Poser (1990) ni hali ya kupotea kwa shadda katika nomino za Kijapani zinazoundwa kutoka kwa vitenzi. Maarifa kutokana na ukiukaji wa Kanuni ya Kufuta Mabano katika nomino za Kijapani zilizoundwa kutoka kwa vitenzi yalitufaa kuelewa mchakato mzima wa fonolojia kurejelea maumbo ya ndani ya neno na namna nomino ambatani hizi huwekwa upya wa shadda. Jedwali 3 lifuatalo linadhihirisha namna shadda huwekwa upya katika nomino ambatani za Kiswahili.
- Mwambatano: m'kazam'jomba

- Uwekaji shadda upya: mkazam'jomba

Kielelezo 2 kinadhihirisha utaratibu wa uwekaji upya wa shadda katika nomino ambatani 'mkazamjomba' ambapo katika hatua ya kwanza nomino ambatani hizi bado hazijapachikwa shadda. Katika hatua ya pili kila neno hupachikwa shadda katika mazingira yake na zinapoambatishwa katika hatua ya tatu kila neno huwa na shadda. Hatua ya nne inadhihirisha uwekaji upya wa shadda ambapo shadda msingi huwekwa upya katika silabi ya pili kutoka mwisho ya nomino 'mkazamjomba' kama ilivyojitokeza katika Kielelezo 2.

Kutoa mwanga zaidi kuhusu mabadiliko ya uwekaji upya wa shadda, tulitumia mchoro wa mzunguko wa Mofolojia Leksia kueleza hatua za uwekaji shadda na namna shadda hupachikwa katika nomino ambatani za Kiswahili. Kielelezo 3 kifuatacho kinaonesha mabadiliko ya uwekaji upya wa shadda katika nomino ambatani 'mkazamjomba.'

38 This work is licensed under a Creative Commons Attribution 4.0 International License. 
Kielelezo 3: Mabadiliko ya Mahali pa kuweka Shadda

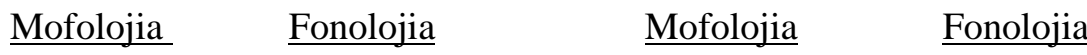

Shina

m'kaza

m'jomba
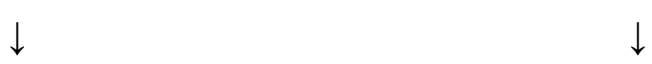

Ngazi $1 \quad[[$ mkaza $]] \leftrightarrow \quad\left[\left[\right.\right.$ m'kaza $^{\prime} \quad[[$ mjomba $]] \leftrightarrow\left[\left[\mathrm{m}^{\prime}\right.\right.$ jomba $\left.]\right]$

Ngazi 2

[[m'kaza]]

$\left[\left[\mathrm{m}^{\prime} \mathrm{jomba}\right]\right]$

(Mwambatano)

$\left[\left[\mathrm{m}^{\prime} \mathrm{kaza}\right][\mathrm{m}\right.$ 'jomba $\left.]\right] \rightarrow$ mkazam'jomba

Kinachotokea katika Kielelezo 3 ni kuwa, kwenye nomino ambatani 'mkazamjomba,' katika mzunguko wa kwanza shadda hupachikwa katika nomino 'mkaza' na 'mjomba' yaani, [[m'kaza]] [[m'jomba]]. Katika mzunguko wa pili mabano ya ndani hufutwa; kwa mujibu wa kanuni ya mzunguko wa mabadiliko, uondoaji wa shadda ya msingi kutoka nomino 'mkaza' na upachikaji upya wa shadda msingi katika nomino 'mjomba' huifanya shadda msingi katika nomino 'mkaza' kuwa hafifu, na hivyo shadda ya msingi huondolewa na kuwekwa upya katika silabi ya pili kutoka mwisho wa nomino 'mjomba' kuwa 'mkazam'jomba.' Chomsky na Halle (1968) wanadai kuwa wakati shadda msingi imewekwa katika nafasi fulani ya neno, basi shadda zingine katika neno hilo huwa hafifu kama inavyojitokeza katika nomino ambatani 'mkazamjomba.' Uondoaji wa shadda kutoka neno la kwanza na upachikaji upya wa shadda katika neno la pili katika mchakato wa Fonolojia ya Kimzunguko unadhihirisha kuwa hakuna ukiukaji wa KKM kwa sababu sheria huona hili kama neno moja, si mawili kama awali. Uondoaji wa shadda msingi katika neno la awali hautokei katika nomino ambatani za Kiswahili pekee bali katika lugha zingine kama Kihispania.

Halle, Harris na Vergnaud (1991) wanaeleza jinsi shadda huwekwa katika nomino ambatani za Kihispania kwa kuongozwa na mkabala wa kufuta shadda hasa nomino ambatani zinapoondoa shadda msingi kutoka mkono wa kulia wa kiambajengo cha nomino ambatani na kuwekwa upya katika mkono wa kushoto wa kiambajengo cha nomino ambatani husika. Mathalan kama inavyojitokeza katika nomino ambatani ya Kihispania 'agwani'eve 'theluji' ambapo shadda ya msingi huondolewa kutoka shina 'agwa na kuwekwa upya katika nomino ni'eve kuwa agwani'eve. Hali hii ya uondoaji wa shadda msingi kutoka neno la kwanza inadhihirika katika nomino ambatani tulizoorodhesha katika Jedwali 3. Odden (2019) anadai kuwa Kanuni ya Kufuta Mabano hukiukwa katika uwekaji wa toni katika vitenzi vya Kikuria hasa sheria ya kuondolewa kwa shina inapokuwa wazi kwa mabano ya ndani ya shina kama inavyojitokeza katika vitenzi tuùre na gi - tùùre kuashiria 'nimechukua hicho.' Hali hii hutokea wakati toni huondolewa kutoka kiambishi na kuwekwa upya katika shina la kitenzi cha Kikuria na hivyo basi, hukiuka Kanuni ya Kufuta Mabano ambayo hairuhusu fonolojia kurejelea mofolojia ya ndani ya neno.

Massamba (2011) anaita aina hii ya mkazo unaoondolewa kutoka neno la kwanza na kupachikwa upya katika neno la pili kuwa mkazo msingi na anaendelea kusema kuwa neno la kwanza kwa kiasi fulani katika nomino ambatani huwa limebakia na mkazo, lakini mkazo ule hauwi mkazo msingi; mkazo huo unaweza kuwa wa pili au tatu kutegemea maneno yanayounda nomino ambatani husika.

Orgun na Inkelas (2002) wanaeleza pia kuwepo kwa changamoto za matumizi ya Kanuni ya Kufuta Mabano katika uambishaji wa vitenzi vya Kiingereza ambapo walibaini baadhi ya vitenzi kuwa na viambishi awali vinavyoonekana na viambishi vya nje. Orgun na Inkelas (2002) wanaendelea kusema kuwa kiambishi cha mofimu huwa wazi kwa uwepo wa kiini cha mofimu nyingine maalumu, yaani aina hizi za vitenzi

39 | This work is licensed under a Creative Commons Attribution 4.0 International License. 
huhitaji muundo wa ndani wa shina kuonekana na mofimu ya nje. Hali hii ya muundo wa ndani kuonekana na mofimu ya nje ulibainika katika nomino ambatani za Kiswahili zinazokiuka Kanuni ya Kufuta Mabano, hasa mofimu za pili zinapochukua viambishi vya wingi. Mathalani tutatumia nomino ambatani 'mwanamke' kama kiwakilishi cha nomino tulizoorodhesha katika Jedwali 3 kuonesha namna muundo wa ndani huonekana na mofimu ya nje. Nomino ambatani 'mwanamke' ina mofu mbili; $\{m w\}$ na \{anamke $\}$ na katika kuambisha nomino ambatani hii kisarufi katika hali ya wingi, mofu ya kwanza $\{m w\}$ na mofu ya pili \{anamke\} huchukua viambishi vya wingi na kuwa $\{w\}$ na \{anawake\}. Hali hii huonesha namna kiambishi $\{m\}$ cha nomino 'mke' katika mofu ya pili \{anamke\} huonekana na mofu ya kwanza $\{m w\}$ na hii ndiyo sababu ya nomino ambatani katika Jedwali 3 hukiuka Kanuni ya Kufuta Mabano.

\section{Hitimisho}

Makala hii imeshughulikia nomino ambatani za Kiswahili zinazokiuka Kanuni ya Kufuta Mabano. Ili kufikia lengo la kudhihirisha nomino ambatani za Kiswahili zinazokiuka Kanuni ya Kufuta Mabano tuliongozwa na mhimili wa Kanuni ya Kufuta Mabano kueleza utaratibu unaohusika katika uundaji wa nomino ambatani na namna ambavyo nomino ambatani hizi hukiuka utaratibu huu hasa pale zinapoambishwa kisarufi katika wingi, hali ambayo husababisha mabadiliko katikati mwa neno baada ya vipashio mbalimbali kuunganishwa na mabano kubomolewa. Makala hii kwa muhtasari imeshughulika na kueleza jinsi baadhi ya nomino ambatani za Kiswahili hukiuka Kanuni ya Kufuta Mabano kwa kuchukua utaratibu huu [wingi $+\mathrm{Y}_{1}$ ] + [wingi $+\mathrm{Y}_{\mathrm{n}}$ ] mathalan; mkazamwana $\rightarrow$ wakazawana ambao ni kinyume cha Kanuni ya Kufuta Mabano kwani kanuni hii hairuhusu aina ya urejeleaji nyuma baada ya mabano kufutwa, na iwapo kutakuwa na urejeleaji nyuma hufuata utaratibu huu [wingi $\left.+\mathrm{Y}_{1}\right]+\left[\mathrm{Y}_{\mathrm{n}}\right]$ kama inavyojitokeza katika nomino ambata; $\boldsymbol{m}$ wanamgambo $\rightarrow$ wanamgambo. Mwisho tulibaini uondoaji wa shadda msingi kutoka neno la kwanza la nomino ambatani na uwekaji upya wa shadda ya msingi katika silabi ya pili kutoka mwisho wa nomino ambatani husika.

\section{Marejeleo}

Allen, M. R. (1978). Morphological Investigations (Unpublished Doctoral, Dissertation). University of Connecticut, Storrs, CT.

BAKITA. (2015). Kamusi Kии уa Kiswahili. Nairobi: Longhorn Press.

Booij, G. (1997). Non- Derivational Phonology Meets Lexical Phonology. In I. Roca (ed.), Derivations and Constraits in Phonology. Oxford: Clarendon Press, 266-88.

Buberwa, A. (2011). Muundo wa Majina ya Mahali katika Kiswahili: Uchunguzi Kifani wa Majina ya Vituo vya Daladala Jijini Dar es Salaam. Kioo cha Lugha, 9(1)1-14.

Buberwa, A. (2017). Ruwaza za Kimofolojia za Majina ya Mahali ya Kihaya: Mtazamo wa Mofolojia Leksika. Kiswahili, 77(1).

Chomsky, N., \& Halle, M. (1968). The Sound Pattern of English. New York: Harper \& Row, Publishers.

Cole, J. (1995). Eliminating Cyclicity as a Source of Complexity in Phonology. In Cole, J., Green. G., \& James, M. (eds.), Linguistics and Computation. Stanford: CSLI, 255-280.

Gichuru, T. M. (2010). Uchanganuzi wa Nomino Ambatani za Kiswahili: Mtazamo wa Mofolojia Leksia (Tasnifu ya Uzamili, haijachapishwa). Chuo Kikuu cha Kenyatta.

Gituru, M. W. (2019). Unyambulishaji wa Vitenzi Katika Kigĩchũgũ (Tasnifu ya Uzamili, haijachapishwa). Chuo Kikuu cha Chuka.

Gordian, E. (2010). The Handling of Morphological Structures in Bantu Lexicography: The Case of Orunyambo Compound Words (Shahada ya Umahiri, haijachapishwa). Chuo Kikuu cha Dar es Salaam.

Habwe, J. \& Karanja, P. (2004). Misingi ya Sarufi ya Kiswahili. Nairobi: Phoenix Publishers.

Halle, M., Harris, J. W., \& Vergnaud, J. R. (1991). A Reexamination of the Stress Erasure

40 | This work is licensed under a Creative Commons Attribution 4.0 International License. 
Convention and Spanish Stress. Linguistic Inquiry, 22(1), 141-159.

Haspelmath, M., \& Sims, A. D (2013). Understanding morphology. New York: Routledge.

Inkelas, S. (1990). Prosodic Constituency in the Lexicon. New York: Garland.

Katamba, F., \& Stonham, J. (2019). Morphology. New York: Palgrave.

Katikiro, E. G. (2018). Tathmini ya Maana katika Maneno Ambatani ya Kiswahili. Mulika Journal, 36(1).

Kiparsky, P. (1982). 'From Cyclic To Lexical Phonology' in Verder Hulst and Smith (eds). The Structure of Phological Representation. Dordrecht: Foris Publication.

Kothari, C. R. (2004) Research Methodology: Methods and Techniques ( $2^{\text {rd }}$ ed). New Delhi: New Age International publishers.

Massamba, D. P. B. (2004). Kamusi ya Isimu na Falsafa ya Lugha. Dar es Salaam: TUKI.

Massamba, D. P. B. (2011). Maendeleo katika Nadharia ya Fonolojia. Dar es Salaam: TATAKI.

Matinde, R. S. (2012). Dafina ya Lugha na Isimu na Nadharia. Kwa Sekondari, Vyиo vya Kati na Vyuo Vikuu. Mwanza: Serengeti Educational Publishers.

McMahon, A. (2000) Lexical Phonology and History of English. Cambridge: Cambridge University Press.

Mgullu, R. S. (1999). Mtaala wa Isimu. Fonetiki, Fonolojia na Mofolojia ya Kiswahili. Nairobi: Longhorn Publishers.

Milroy, A. L. (1987). Observing and Analysing Natural Languages. Oxford: Basil Blackwell Ltd.

Mohamed, S. A. (2010). Nyuso za Mwanamke. Nairobi: Longhorn Publishers Limited.
Mohanan, K. P. (1986). The Theory of Lexical Phonology. Dordrecht: Reidel.

Mustafa, S. K. (2020). Categorization of Compound Nouns in Kurdish and English. LLT Journal: A Journal on Language and Language Teaching, 23(1), 104-115.

Odden, D. (2019). Predicting Tone in Kikuria. Publications in African Languages and Linguistics, 311-326, doi: 10.1515/9783110882681-02.

Orgun, C. O., \& Inkelas, S. (2002). Reconsidering Bracket Erasure. In Yearbook of Morphology 2001 (115-146).

Pesetsky, D. (1979). Russian morphology and lexical theory. Manuscript, MIT.

Poser, W. J. (1990). The Phonetics and Phonology of Tone and Intonation in Japanese. Journal of Japanese Linguistics, 12(1), 208-209.

Prince, A., \& Smolensky, P. (1993). Optimality Theory: Constraint Interaction in Generative Grammar (Unpublished, Ms). Rutgers University.

Rubanza, Y. I. (1996). Mofolojia ya Kiswahili. Dar es Salaam: Chuo Kikuu Huria cha Tanzania.

Siegel, D. (1974). Topics in English Morphology (Unpublished Doctoral, Dissertation). MIT, Cambridge, Massachusetts.

TUKI (2004). Kamusi ya Kiswahili Sanifu. Dar es Salaam: Oxford University Press.

41 This work is licensed under a Creative Commons Attribution 4.0 International License. 